\title{
The UVES Paranal Observatory Project: a public library of high resolution stellar spectra†
}

\author{
E. Jehin ${ }^{1}$, S. Bagnulo ${ }^{1}$, C. Melo ${ }^{1}$, C. Ledoux ${ }^{1}$ and R. Cabanac ${ }^{2}$ \\ ${ }^{1}$ European Southern Observatory, Alonso de Cordova 3107, Santiago 19, Chile \\ email: ejehin@eso.org, sbagnulo@eso.org, cmelo@eso.org, cledoux@eso.org, \\ ${ }^{2}$ Canada-France Hawaii Telescope, 65-1238 Mamalahoa Hwy, Kamuela, HI 96743, USA \\ email: cabanac@cfht.hawaii.edu
}

\begin{abstract}
The UVES Paranal Observatory Project (POP), is an ESO public database of about 400 stars whose high quality spectra were obtained with UVES, the high resolution spectrometer of the VLT. All stars were observed with two instrument modes, in order to cover almost completely the optical region $(300-1000 \mathrm{~nm})$. The resolving power is about 80000 , and for most of the spectra, the typical $\mathrm{S} / \mathrm{N}$ ratio is $300-500$ in the $\mathrm{V}$ band. Program stars fall into two groups, stars belonging to open clusters IC 2391 and NGC 6475, and bright field stars. For field stars, the only selection criterion applied was to cover the largest possible variety of spectral types in the HR diagram, including peculiar objects, e.g., Ap and Bp stars, Wolf-Rayet stars, Be stars, carbon stars and metal poor stars. The spectra have been reduced, coadded and merged and various products can be downloaded from a public area. For each star the final spectrum may be displayed through a dedicated user-friendly Spectral Preview Interface. The database is accessible at http://www.eso.org/uvespop
\end{abstract}

Keywords. astronomical data bases: atlases, stars: general

\section{Introduction}

While at Paranal Observatory (ESO, Chile) twilight time is normally used for calibration of the instruments in operation, on UT 2 it was, in the past, restricted to the observation of UVES spectro-photometric standard stars using only part of the twilight. The UVES POP has been conceived to take advantage of the idle times in the darker part of the twilights in order to build a public library of stellar spectra of general interest through observations of bright stars $(\mathrm{V}<8.5)$.

The POP library is the richest database of observed stellar optical spectral lines and can be seen as an observational counterpart of atomic databases. Its spectra can be used for many different studies: testing model atmospheres and spectral synthesis codes, computing very accurate abundances, looking for interstellar absorption features, carrying out population studies, etc.

\section{The Sample \& Instrument Setup}

The POP sample covers a large variety of spectral types in the HR diagram, including peculiar objects, e.g., Ap and Bp stars, Wolf-Rayet stars, Be stars, carbon and metal poor

$\dagger$ Based on observations collected at the European Southern Observatory, Paranal, Chile (Program ID: 266.D-5655) 
stars. A total of about 300 field stars were observed in addition to 50 and 30 probable members of, respectively, IC 2391 and NGC 6475.

The observations were carried out using the red and blue arms of UVES simultaneously. Thanks to the use of two dichroic beam splitters, the complete visible range (304-1040nm) could be covered (except for a few small gaps) in only two exposures. Note that a minimum of two spectra were taken per setting but usually many more. The slit width was set to $0.5^{\prime \prime}$ which provides a resolving power of about 80000 .

\section{Data Reduction \& Data Quality Control}

The data were reduced using a modified and fine tuned version of the UVES context v1.2 of MIDAS and a dedicated data organizer to deal with the huge amount of data in an automatized way. Due to the high $\mathrm{S} / \mathrm{N}$ of the observations $(\mathrm{S} / \mathrm{N}>100)$ the average extraction was preferred over the optimum extraction algorithm. An automatic procedure was developed to convert the wavelength scale of the spectra to heliocentric rest-frame, average multiple individual exposures and connect the spectra from different instrument settings all together. All individual spectra were extinction-corrected and a relative flux calibration applied using the UVES master response curves provided by ESO. The final result is one single spectrum per star covering the whole optical range from about 304 to $1040 \mathrm{~nm}$ and with the correct physical energy distribution (but not with the correct absolute flux scale due to slit losses) and with a typical $\mathrm{S} / \mathrm{N} \sim 400$ in the V-band.

Quality control was carried out by our team on the hundreds of individual spectra and reported in a dedicated logfile. Possible problems are saturated frames, failure to extract the spectra, low quality of the spectral order merging, etc.

\section{Data Release \& Spectrum Preview Interface}

The data are released through an ESO public Web page : http://www.eso.org/uvespop. For each star, the reduced data are available in the form of seven gzipped tar files plus one fits table. Six of these tar files correspond to the observed setups: 346B, 437B, 580L, $580 \mathrm{U}, 860 \mathrm{~L}$ and $860 \mathrm{U}$. Each tar file contains all individual reduced spectra and associated variance spectra, order by order (unmerged) reduced spectra, plus sky spectra. A fits table (also available in ascii) is generated by averaging and connecting all final pipeline products. This final spectrum has the correct star's spectral energy distribution.

A dedicated navigation tool has been developed to allow a friendly access and query of the database and to plot and retrieve the final spectra under several formats.

For more information about the UVES POP please refer to:

\section{References}

Bagnulo, S., Jehin, E., Ledoux, C., Cabanac, R., Melo, C., \& Gilmozzi, R. 2003, The ESO Messenger 114, 10 